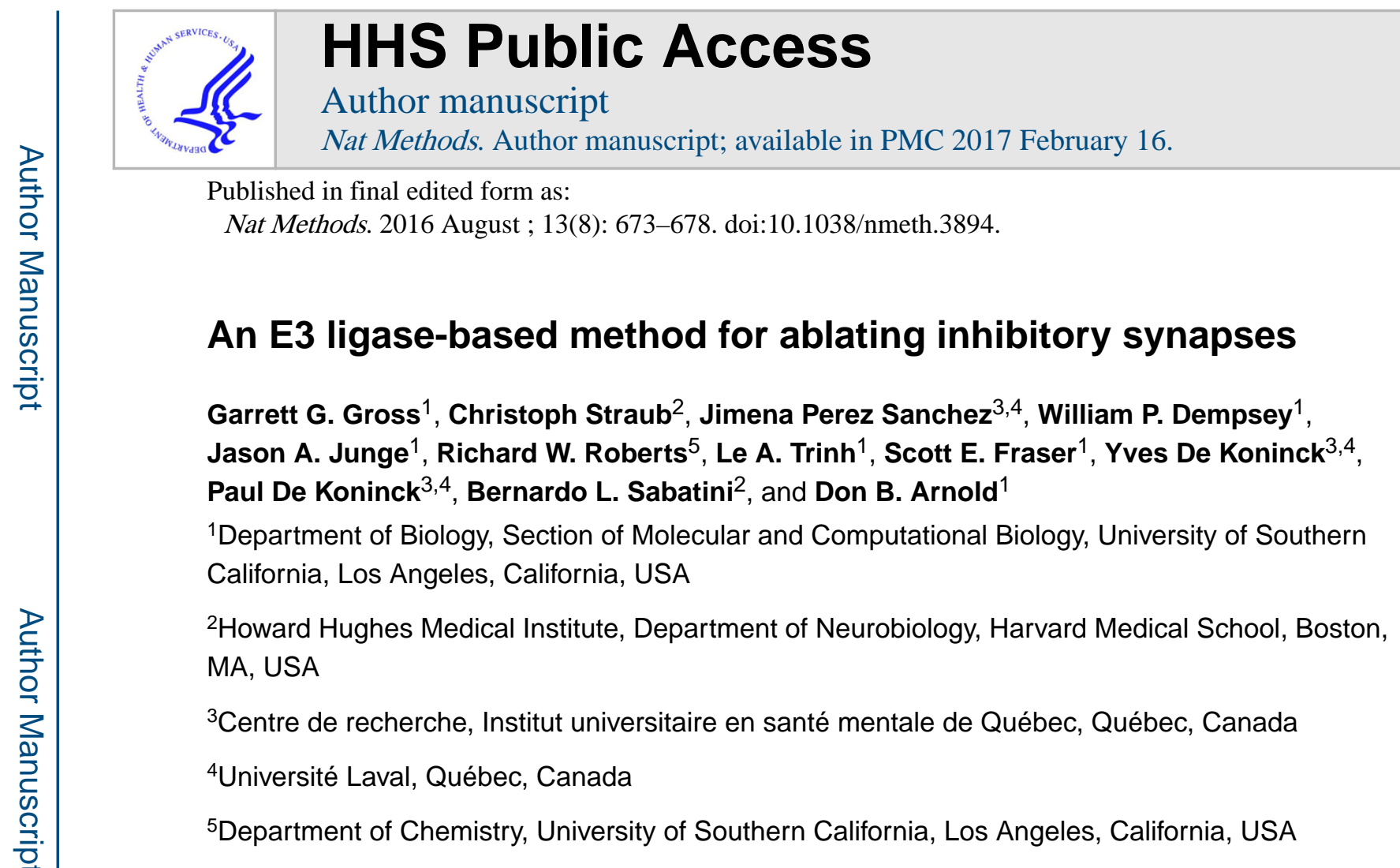

\title{
Abstract
}

Although neuronal activity can be modulated using a variety of techniques, there are currently few methods for controlling neuronal connectivity. We introduce a tool (GFE3) that mediates the fast, specific and reversible elimination of inhibitory synaptic inputs onto genetically determined neurons. GFE3 is a fusion between an E3 ligase, which mediates the ubiquitination and rapid degradation of proteins, and a recombinant, antibody-like protein (FingR) that binds to Gephyrin. Expression of GFE3 leads to a strong and specific reduction of Gephyrin in culture or in vivo and to a substantial decrease in phasic inhibition onto cells that express GFE3. By temporarily expressing GFE3 we showed that inhibitory synapses regrow following ablation. Thus, we have created a simple, reversible method for modulating inhibitory synaptic input onto genetically determined cells.

\section{Editorial summary}

When studying neural circuitry, the ablation of synapses may be an alternative to optogenetic manipulation of neurons. Arnold et al. report a genetically encoded tool called GFE3 that eliminates inhibitory inputs into neurons expressing GFE3.

\footnotetext{
Corresponding author: Don B. Arnold darnold@usc.edu. Author Contributions:

G.G.G. and D.B.A conceived of the project and designed the imaging, cell biology and biochemistry experiments, which were performed by G.G.G. C.S. and B.L.S. designed and C.S. performed electrophysiology experiments in slices. J.P.S., P.D.K, and Y.D.K. designed and J.P.S. performed electrophysiology experiments on dissociated neurons. W.P.D and D.B.A designed and W.P.D. performed experiments on zebrafish assisted by L.A.T and S.E.F. J.A.J. and R.W.R. contributed to the design of constructs. D.B.A. and G.G.G wrote the paper with contributions from all authors.

Competing Financial Interest Statement

The authors declare no competing financial interests.
} 
We sought to create a method for eliminating inhibitory inputs onto neurons by ablating Gephyrin, a postsynaptic protein that clusters $\mathrm{GABA}_{\mathrm{A}}$ and Glycine receptors ${ }^{1-3}$. Traditional methods for eliminating protein expression, such as gene deletion ${ }^{4}$ or siRNA ${ }^{5}$, work by means of cutting off protein supply, and thus become effective only as the protein degrades, which can take a week or longer ${ }^{6,7}$. Furthermore, they are not easily reversible. Therefore, our approach uses an E3 Ligase enzyme, which marks proteins for degradation by mediating the covalent attachment of ubiquitin polypeptides to lysine residues, causing the marked proteins to be transported to the proteasome and degraded ${ }^{8}$. E3 ligases can be engineered to have arbitrary specificity by fusing their ubiquitin transferase domains with proteins that bind to specific targets. For instance, a fusion of an E3 ligase with a GFP nanobody degrades exogenously expressed GFP in vivo ${ }^{9}$. However, endogenous proteins have been targeted with genetically encoded molecules in few examples only ${ }^{10}$.

We have generated a protein, GFE3, that mediates the fast and specific degradation of Gephyrin, eliminating the inhibitory synaptic inputs to the neurons in which it is expressed. Gephyrin degradation is complete within 5 hours of inducing GFE3 expression, and cessation of GFE3 expression results in inhibitory synapses reappearing within 48 hours. Thus, GFE3 can specifically and reversibly alter synaptic connectivity.

\section{Results}

\section{GFE3 mediates degradation of Gephyrin}

We generated GFE3 by fusing GFP-GPHN.FingR, an antibody-like protein based on the 10FNIII domain of fibronectin that binds Gephyrin with high affinity ${ }^{11}$, with the RING domain of XIAP, an E3 ubiquitin ligase ${ }^{12}$ (Supplementary Fig. 1). To test whether GFE3 can mediate the elimination of endogenous Gephyrin, we compared the expression of synaptic proteins from cultures of cortical neurons expressing GFE3 and cultures expressing control constructs. Neurons that expressed either GPHN.FingR only or a fusion of the RING domain with a FingR containing random residues within its target binding domains (RandE3) displayed both Gephyrin and PSD-95 puncta, whereas neurons expressing GFE3 displayed PSD-95 puncta, but no Gephyrin puncta (Fig. 1a-c, Supplementary Fig. 1).

To investigate GFE3's mode of action, we co-expressed GFE3, GPHN.FingR or RandE3 in COS-7 cells with Gephyrin-GFP. In the course of 24 hours, GFE3 reduced exogenous Gephyrin-GFP by $\sim 80 \%$ compared to the controls. This reduction was dependent on proteasome function as shown by treatment with Lactacystin (clasto-Lactacystin-[beta]lactone), a highly specific and irreversible proteasome inhibitor (Supplementary Fig. 1). We also co-expressed Gephyrin-GFP in COS-7 cells with HA-Ubiquitin and either GFE3, GPHN.FingR or RandE3 in the presence of Lactacystin and immunoprecipitated with an anti-Gephyrin antibody. Anti-HA staining of the precipitates revealed a prominent high molecular weight band in the GFE3 lane that likely corresponds to poly-ubiquitinated Gephyrin (Supplementary Fig. 1). Thus, our results are consistent with GFE3 causing polyubiquitination of Gephyrin followed by degradation in the proteasome.

To quantify the effect of expressing GFE3 in neurons, we compared uninfected (control) neuronal cultures to cultures infected with a lentivirus encoding either GFE3, GPHN.FingR 
or RandE3. Lentiviral infection led to efficient expression of GFE3 and a sharp reduction of endogenous Gephyrin, whereas infection with RandE3 or GPHN.FingR, while equally efficient, did not affect Gephyrin expression (Supplementary Fig. 2). GFE3 expressing cultures expressed 80\% less Gephyrin compared with neurons expressing GPHN.FingR (Fig. 1d, Supplementary Fig. 3). Despite this effect on Gephyrin expression, GFE3 had a negligible effect on expression levels of the $\mathrm{GABA}_{\mathrm{A}}$ receptor, PSD-95, GluA1, GluN2B, or the Gephyrin interactors Collybistin $\mathrm{II}^{13}$ and GABARAP ${ }^{14}$ (Fig. 1d). Indeed, expression levels of these proteins are not significantly different in cells expressing GFE3 versus the three types of control cells (Supplementary Fig. 4). Thus, expression of GFE3 leads to the efficient, specific reduction of endogenous Gephyrin levels.

Because Gephyrin clusters $\mathrm{GABA}_{\mathrm{A}}$ receptors, we examined the effect of GFE3 on the receptors' distribution. In control cells $\mathrm{GABA}_{\mathrm{A}}$ receptors were clustered and colocalized with Gephyrin, whereas in neurons transfected with GFE3 $\mathrm{GABA}_{\mathrm{A}}$ receptors were diffusely distributed and Gephyrin staining was not visible (Fig. 1e, f; Supplementary Fig. 4). Thus, reducing endogenous Gephyrin by expressing GFE3 appears to release $\mathrm{GABA}_{\mathrm{A}}$ receptors from postsynaptic clusters, allowing them to distribute through the dendrites, but does not substantially reduce their expression, consistent with results obtained with siRNA against Gephyrin ${ }^{15}$. Furthermore, although expression of GFE3 causes a large change in Gephyrin expression levels, its expression is not associated with changes in either cell viability or overall proteasome activity (Supplementary Fig. 5).

\section{GFE3 expression eliminates phasic inhibition}

To examine the functional consequences of GFE3 expression, we transfected cultured hippocampal neurons with GFE3, GFP (control) or RandE3. We recorded from neurons with access resistance below $30 \mathrm{M} \Omega$ that varied by $20 \%$ or less using whole cell patch clamp. GFE3-expressing cells exhibited miniature inhibitory postsynaptic currents (mIPSCs) with a roughly $65 \%$ decrease in the frequency and $30 \%$ reduction in amplitude when compared to control cells or to cells expressing RandE3 (Fig. 2a-d). In contrast, miniature EPSCs recorded under similar conditions in cells expressing GFE3 showed no significant difference in either frequency or amplitude when compared with control cells $(P>0.75$, Mann Whitney; Supplementary Fig. 6). To test whether functional $\mathrm{GABA}_{\mathrm{A}}$ receptors are still present on the neuronal membrane in cells expressing GFE3, we applied a high concentration of GABA $(1 \mathrm{mM})$ to the bath. Under these conditions the amplitude of evoked currents was not significantly different between cells expressing GFE3 and controls $(P>0.5$, Mann-Whitney; Fig. 2e, f). This suggests that unclustered $\mathrm{GABA}_{\mathrm{A}}$ receptors are on the cell surface and remain functional. Furthermore, noise analysis of these data is consistent with the kinetics of $\mathrm{GABA}_{\mathrm{A}}$ receptors being similar in the two conditions (Supplementary Fig. 7).

To test the efficiency of GFE3 in abolishing GABAergic synaptic transmission in the intact brain, we virally infected indirect spiny projection neurons (iSPNs) to induce GFE3 or GPHN.FingR expression in a cre-dependent manner in the dorsal striatum of Adora2A-cre mice, while simultaneously expressing Channelrhodopsin2 (ChR2) in non-cre-expressing striatal cells (Fig. 3a). This approach resulted in light-evoked synaptic GABAergic transmission onto iSPNs, with GABA being released presumably mostly from direct SPNs, 
with some contribution from GABAergic interneurons ${ }^{16}$. A single light pulse reliably evoked $\mathrm{GABA}_{\mathrm{A}}$ receptor mediated synaptic currents under control conditions where we expressed GPHN.FingR, but currents were abolished in iSPNs expressing GFE3 (Fig. 3b). We also examined spontaneous IPSCs, and found a significant reduction in both frequency ( $P=0.0008$, Mann-Whitney) and amplitude ( $P<0.03$, Mann-Whitney), compared to control conditions (Fig. 3c). This reduction in spontaneous synaptic activity was selective for GABAergic transmission, as spontaneous excitatory inputs were unchanged (Fig. 3c). Thus, expression of GFE3 specifically eliminates functional GABAergic synapses, without affecting glutamatergic synapses. In contrast, the amplitude of tonic GABA currents ${ }^{17-19}$ showed a tendency towards reduction, but this change did not rise to the level of significance (Fig. 3d, e). These results indicate that while there might possibly be a partial reduction in total (synaptic and extra-synaptic) $\mathrm{GABA}_{\mathrm{A}}$ receptor expression at the cell surface, elimination of $\mathrm{GABA}_{\mathrm{A}}$ receptors from the synapse by GFE3 is not due to a general elimination of $\mathrm{GABA}_{\mathrm{A}}$ receptors from the cell, consistent with our results in dissociated cultures (Figs. 1e, f; 2e, f). Thus, our results are consistent with GFE3 specifically eliminating the phasic inhibitory input onto neurons in the intact brain.

\section{GFE3 in zebrafish spinal neurons causes motor defects}

To further test the efficacy of GFE3 in vivo, we injected zebrafish zygotes with a plasmid containing GFE3-GFP driven by the $m n x 1$ enhancer, which restricts expression to spinal cord neurons ${ }^{20}$. Expression of GFE3-GFP was both efficient and specific as indicated by numerous labeled cells along the midline in the spinal cord 2 days post-injection (Fig. 4a). In fish expressing RandE3-GFP, GFP is visible within the cell bodies of spinal cord neurons, while at the outer edge of these cell bodies there is a ring of Gephyrin staining (Fig. 4b). In contrast, Gephyrin staining is sharply reduced in the cell bodies of spinal cord neurons expressing GFE3-GFP (Fig. 4c). Discontinuous staining on the edge of the GFP staining likely arises from unlabeled neighboring cells. Furthermore, the average level of Gephyrin staining surrounding the cell bodies of cells expressing GFE3-GFP was significantly lower than that of cells expressing RandE3-GFP alone ( $P<0.008$, Mann-Whitney; Fig. 4d).

A major role of inhibitory neurons in the zebrafish spinal cord is to prevent co-contractions of opposing muscles by inhibiting contralateral motoneurons ${ }^{21}$. Accordingly, zebrafish expressing mutant Glycine receptors in the spinal cord that fail to cluster at inhibitory synapses exhibit co-contraction of opposing muscles within the tail ${ }^{22,23}$. Zebrafish expressing GFE3-GFP (Supplementary Videos 1,2) exhibited a lack of coordination when compared to zebrafish expressing RandE3-GFP (Supplementary Videos 3, 4), which would be expected if the lack of Gephyrin caused co-contraction of opposing muscles. To quantify these effects we took advantage of a peculiarity of the stereotyped tail flick behavior ${ }^{24,25}$. Fish expressing RandE3-GFP bend their tails in a tight circle so that the tip of the tail touches the base of the tail (Fig. 4e, Supplementary Fig. 8), where it connects with the abdomen of the fish. In contrast, fish that express GFE3-GFP do not touch the bases of their tails with the tips (Fig. 4f, Supplementary Fig. 8). Indeed, the minimum distance between the base and the tip of the tail during 5 tail flicks in zebrafish expressing GFE3-GFP was more than twice that of the same distance measured in zebrafish expressing RandE3-GFP (Fig. 4g). These results are consistent with expression of GFE3 causing the efficient ablation 
of Gephyrin in vivo, resulting in the loss of phasic inhibition within spinal cord neurons in zebrafish.

\section{GFE3-mediated loss of Gephyrin is reversible}

An advantage of the GFE3 approach is that Gephyrin expression is modified at the level of protein degradation, and thus the level of Gephyrin is expected to respond quickly to the expression or elimination of GFE3. To test this hypothesis, we expressed GPHN.FingR-GFP along with doxycycline(dox)-dependent GFE3-TagRFP in cortical neurons in culture and incubated for 4-7 days without dox, labeling inhibitory synapses with GPHN.FingR-GFP. We then induced expression of GFE3-TagRFP using dox (Fig. 5a, Supplementary Fig. 9), and found efficient elimination of Gephyrin in less than 5 hours as indicated by the absence of GPHN.FingR-GFP labeling (Fig 5a, b). Fixing the same cells and immunostaining for endogenous Gephyrin (green) and GFE3-TagRFP (red) confirmed that endogenous Gephyrin had been eliminated (Fig. 5c). In contrast, transfection of siRNA led to an $80 \%$ reduction in the number of Gephyrin puncta after 5 days in dissociated hippocampal cultures ${ }^{26}$.

To demonstrate reversible elimination of Gephyrin expression, we added dox to neurons transfected with GPHN.FingR-GFP and dox-dependent GFE3-TagRFP for 24 hours, and then removed dox and waited 48 hours to determine whether the Gephyrin puncta would return (Supplementary Fig. 9). As in the previous experiment, Gephyrin puncta labeled with GPHN.FingR-GFP disappeared following 24 hours of dox-induced GFE3-TagRFP expression (Fig. 5d, e; Supplementary Fig. 9). Removal of dox for 48 hours resulted in a reappearance of Gephyrin puncta (Fig. 5f), many in places that are close to their original locations (Fig. 5g). Gephyrin immunostaining at 48 hours after removal of Dox showed that newly formed Gephyrin puncta co-localized with staining for presynaptic GAD-65 (Fig. 5h), suggesting that the recovered Gephyrin puncta have corresponding presynaptic sites, and thus are likely part of reconstituted synapses. Finally, quantitation of GPHN.FingR-GFP shows that following recovery total FingR staining in puncta is present at levels that are comparable to initial levels (Fig. 5i). Thus, by controlling the expression of GFE3 it is possible to induce the reversible ablation of Gephyrin.

\section{Discussion}

FingRs can target fluorescent proteins to endogenous target proteins, allowing the targets and the macromolecular structures where they reside to be visualized in vivo ${ }^{11,27}$. Here we show that an E3 ligase can also be targeted specifically using a FingR, causing expression of its target protein to be quickly and reversibly eliminated. Although this is not the first method developed for direct elimination of protein expression, it differs in important respects from previously described methods. Many such methods are specific to exogenously expressed proteins ${ }^{9,28}$, or are not genetically encoded ${ }^{29}$. Furthermore, FingRs bind to their targets without affecting target function or expression ${ }^{11}$, which means that protein elimination is entirely due to increased degradation through the E3 ligase and the Ubiquitin proteasome system. This is distinct from scFv-based proteins that reduce Gephyrin expression through an unknown mechanism, with unknown off-target effects ${ }^{30}$. In addition, because the Gephyrin FingR was specifically engineered to bind Gephyrin with 
very high affinity and specificity, the fidelity with which it degrades its target is likely to be much higher than that of a system that depends on naturally occurring protein-protein interactions, such as those involving PDZ domains, which have relatively low affinity and specificity ${ }^{31,32}$. Furthermore, positively charged cell-permeant peptides such as Tat, upon which systems mediating degradation with peptides rely, are inefficient, unstable, and induce cytotoxicity ${ }^{33}$.

E3 ligases can be fused to FingRs targeted against other proteins, suggesting that our approach could be generalizable for reversibly ablating a wide range of endogenous targets. In addition, because of its modular nature, we envision that GFE3 function could be engineered to rely on light dependent association of the two domains, making the complex active in response to light.

Modulating the excitability of neurons using optogenetics has revealed the electrophysiological properties of specific neuronal cell types and their roles in circuits and functional networks, which was not possible with previous pharmacological approaches. Like optogenetic proteins, GFE3 can be expressed in specific cell types. However, it acts at a qualitatively different level than optogenetics - modifying endogenous synaptic connectivity rather than directly inducing the opening of exogenous ion channels or the activation of pumps ${ }^{34}$. GFE3 also differs from AS-PaRac-1, which can inducibly reduce the strength of excitatory synapses, in that GFE3 eliminates Gephyrin, a component of the synapse, whereas AS-PaRac1 causes changes in the actin cytoskeleton supporting dendritic spines by modulating function of the $\mathrm{G}$ protein $\operatorname{Rac} 1^{35}$.

By targeting expression of GFE3 to excitatory cells it should increase excitability within a specific brain region, whereas expressing it in inhibitory cells should decrease excitability. Thus, it should be possible to probe the effects of systematically modulating the excitability of specific regions of the brain, both in wild-type animals and in disease models in a way that does not directly interfere with the firing patterns of cells.

In conclusion, GFE3 provides a fast, specific and reversible method for modulating inhibition onto defined cells. Furthermore, it provides a framework for generating a diverse array of genetically encoded modulators of expression and function that act specifically on virtually any target protein.

\section{Online Methods}

\section{cDNA Constructs}

The Gephyrin (GFE3) and Random (RandE3) ablating FingRs were constructed by fusing a DNA sequence encoding three consecutive HA-tags followed by the amino acids 440-496 of the RING domain of rat XIAP 3' to the Gephyrin FingR gene ${ }^{11}$ or to the Random FingR gene FN04 ${ }^{36}$ (Supplementary Fig. 1). In addition, the gene for EGFP or TagRFP (Evrogen, cat. \# FP141) was linked 3' to a DNA spacer encoding the amino acid sequence GGGS repeated four times, which was then fused 5' to the Gephyrin FingR to allow for direct visualization of the GFE3 in living cells. Expression constructs were made by ligating these genes into the pCAG, pFUW or $\mathrm{pAAV}^{37}$ expression vector. The construction of 
transcriptionally controlled Gephyrin.FingR-GFP-CCR5ZFL-KRAB(A) (Addgene, cat. \# 46296) and Gephyrin-GFP were described previously ${ }^{11}$. Note that in COS-7 cell experiments a non-transcriptionally controlled version of GPHN.FingR was used, but transcriptionally controlled GPHN.FingR was used in all other contexts. GHA-tagged Human Ubiquitin inserted into the constitutive mammalian expression vector pcDNA3 (Addgene plasmid \# 18712) ${ }^{38}$. Plasmids for expression of RandE3 or GFE3 in zebrafish were created by ligating the assembled gene (RandE3 or GFE3) into the modified expression vector $\mathrm{pMT}(3 m n x 1)$, which contains a triplicated enhancer element from the $m n x 1$ gene upstream of the MCS that drives expression of the ablating FingR in spinal cord neurons ${ }^{20}$. A vector to mediate inducible expression of GFE3 (GFE3-TagRFP ind $_{\text {) }}$ was generated by subcloning tagRFP-4(GGGS)-GFE3 into the pTRE-3G vector (Tet-On 3G, Clontech, cat. \# 631337). GFE3, RandE3, or GPHN.FingR was inserted into the MCS of the pFUW vector for production of lentivirus at the USC School of Pharmacy Lentiviral Laboratory. Similarly, GFE3 or GPHN.FingR was subcloned into the 'cre-on' pAAV-EF1a-DIO vector for production of AAV at the University of Pennsylvania School of Medicine Vector Core. The sequences for all constructs were verified by DNA sequencing.

\section{Expression of Gephyrin Ablating FingRs in Cells}

Constitutive expression of GPHN.FingR, GFE3 or RandE3 in mammalian cells was achieved through CalPhos (Clontech, cat. \# 631312) or Lipofectamine 2000 (Thermo Fisher Scientific, cat. \# 11668019) transfection, lentiviral transduction or adeno-associated virus (AAV) transduction of the construct into cells. Inducible expression of GFE3 was achieved by CalPhos-mediated co-transfection of GFE3-TagRFP ${ }_{\text {ind }}$ with the doxycycline-dependent Tet-On expression vector pCMV-Tet-3G (Tet-On 3G, Clontech, cat. \# 631337) into cells. In experiments where the transcriptionally controlled Gephyrin FingR was co-expressed with doxycycline-induced GFE3, $2 \mu \mathrm{g}$ of Gephyrin.FingR-GFP-CCR5ZFL-KRAB(A) was cotransfected with $1 \mu \mathrm{g}$ each of GFE3-TagRFP ${ }_{\text {ind }}$ and pCMV-Tet-3G into cells. $1 \mu \mathrm{g}$ of doxycycline (EMD Millipore, cat. \# 631311) in DMSO was added per $\mathrm{mL}$ of medium to cells to activate the Tet-On $3 \mathrm{G}$ expression system, and was removed by washing the cells six times with conditioned medium. Expression time of constructs ranged from 5 hours to 10 days, as specified. For lentiviral infection 4 infectious units (IFU) of lentivirus at a titer of $3.0 \times 10^{7} \mathrm{IFU} / \mathrm{mL}$ was used per cell by adding concentrated virus to the medium for 16 to 24 hours. Transfection or transduction of cells was performed according to the manufacturer's suggested protocol. Experimental protocols were conducted according to the US National Institutes of Health guidelines for animal research and were approved by the Institutional Animal Care and Use Committee at the University of Southern California, the Animal Care Committee at Laval University and the Institutional Animal Care and Use Committee at Harvard Medical School.

\section{Ubiquitination and Proteasome Dependency}

COS-7 cells (ATCC, cat. \# CRL-1651) were cultured in Dulbecco's modified eagle's medium (ATCC, cat. \# 30-2002) supplemented with $10 \%$ fetal bovine serum in $5 \% \mathrm{CO}_{2}$ to a confluency of $80 \%$ on a $100 \times 20 \mathrm{~mm}$ tissue culture dish before being co-transfected with constructs constitutively expressing the RandE3, GFE3 or the Gephyrin ablating FingR control along with Gephyrin-GFP and HA-Ubiquitin. 24 hours after transfection, cells were 
lysed in $1 \mathrm{~mL}$ of lysis buffer (150 mM NaCl, $1 \mathrm{mM}$ EDTA, $10 \mathrm{mM}$ Tris, pH 8.0, 1\% NP40, $0.12 \mathrm{mg} / \mathrm{mL}$ PMSF, $2 \mu \mathrm{g} / \mathrm{mL}$ Leupeptin, $1 \mu \mathrm{g} / \mathrm{mL}$ Aprotinin, $10 \mathrm{mM} \mathrm{NaF}, 1 \mu \mathrm{g} / \mathrm{mL}$ Pepstatin). Proteasome activity in cells was arrested by adding the highly specific and irreversible inhibitor clasto-Lactacystin-[beta]-lactone (10 $\mu \mathrm{M}$, VWR, cat. \# 80052-808) in DMSO to the culture medium. To examine Gephyrin ubiquitination in cells, $500 \mu$ of lysate was pre-cleared with agarose beads for 2 hours at $4^{\circ} \mathrm{C}$ and then incubated overnight at $4{ }^{\circ} \mathrm{C}$ with a rabbit anti-Gephyrin antibody (Bethyl Laboratories, cat. \# A304-245A) diluted 1:100. The next day, $50 \mu \mathrm{l}$ of protein A/G agarose beads (Pierce, cat. \# 20421) were added to the lysate. The beads were then nutated at $4{ }^{\circ} \mathrm{C}$ for 8 hours to immunoprecipitate (IP) Gephyrin, washed six times in wash buffer (as lysis buffer, except with $0.1 \%$ NP40) and finally boiled for 5 minutes in Laemmli sample buffer (62.5 mM Tris-HCl, pH 6.8, 2.5\% SDS, $10 \%$ Glycerol, $0.02 \%$ bromophenol blue, 5\% 2-mercaptoethanol). Cell lysate and eluate from the Gephyrin IP were run on pre-cast Any kD SDS-PAGE gels (Bio-Rad, cat. \# 4569033) and transferred to a low fluorescence PVDF membrane (Bio-Rad, cat. \# 1620264). The blots were then probed with a mouse anti-Gephyrin antibody (Synaptic Systems, cat. \# 147 111), 1:1000, a mouse anti-beta-tubulin antibody (Sigma, cat. \# T4026), 1:5000, or a mouse antiHA antibody (Covance/BioLegend, cat. \# 901513), 1:500. Secondary antibody used was goat anti-mouse Alexa Fluor 750 (Thermo Fisher Scientific, cat. \# A-21037), 1:1000. Blots were imaged with the Odyssey Infrared Imaging System (LI-COR Biosciences). Steadystate protein levels were determined using ImageJ software (US National Institute of Health). Prior to analyses of data, Gephyrin-GFP levels were normalized to beta-tubulin levels. Data are represented as mean \pm SEM and were compared using the non-parametric Kruskal-Wallis test. A $P$ value $<0.05$ was considered significant. The supplier (ATCC) tested the cells for mycoplasma and confirmed their species of origin by COI assay.

\section{Lentiviral Transduction Efficiency}

Efficiency of lentiviral transduction with GFE3, RandE3 or GPHN.FingR was assessed by counting the number of GFP positive and negative cells in five $133.3 \mu \mathrm{m}^{2}$ images taken from infected Delta T dishes. In brief, live cell morphology and GFP expression were imaged and analyzed first with brightfield illumination and FITC fluorescence. Cells were then fixed and stained for GFP and Gephyrin, and mounted in Fluoromount-G with DAPI (Electron Microscopy Sciences, cat. \# 17984-24). A GFP positive cell was identified by colocalization of GFP with DAPI in the cell body, whereas a GFP negative cell had no colocalization of DAPI with GFP. Image analyses were done blinded with ImageJ software. Data are represented as mean \pm SEM and were subjected to a Kruskal-Wallis test. A $P$ value $<0.05$ was considered significant.

\section{Quantitation of knockdown of synaptic protein levels following expression of GFE3}

Dissociated rat cortical neurons seeded at a density of $7.5 \times 10^{5}$ on $100 \times 20 \mathrm{~mm}$ tissue culture dishes were transduced with $3.0 \times 10^{6}$ IFUs of lentivirus with the RandE3, GFE3 or GPHN.FingR. After 16 hours of expression, cells were lysed in $1 \mathrm{~mL}$ of lysis buffer with $1 \%$ Triton X-100. Samples were then run on Any kD SDS-PAGE gels and transferred to PVDF membrane. The blots were probed with a mouse anti-Gephyrin antibody, 1:1000, a rabbit anti-GABA ${ }_{A}$ receptor alpha1 antibody (EMD Millipore, cat. \# 06-868), 1:1000, a mouse anti-PSD-95 antibody (NeuroMab, cat. \# 75-028), 1:1000, a mouse anti-GluA1 antibody 
(NeuroMab, cat. \# 75-327), 1:1000, a mouse anti-GluN2B antibody (NeuroMab, cat. \# 75-097), 1:1000, a mouse anti-Collybistin II antibody (Santa Cruz Biotechnology, cat. \# sc-136393), 1:1000, a rabbit anti-GABARAP antibody (Origene, cat. \# TA307845), 1:1000, or a mouse anti-beta-tubulin antibody, 1:5000. Secondary antibody used was goat antimouse Alexa Fluor 750, 1:1000, or goat anti-rabbit Alexa Fluor 750, 1:1000 (Thermo Fisher Scientific, cat. \# A-21039). The blots were imaged at multiple intensities using the Odyssey Infrared Imaging System. Measurements of synaptic protein steady-state levels from four independent experiments were obtained using ImageJ software. This data was normalized to beta-tubulin levels and subjected to a Kruskal-Wallis test. All data are expressed as mean \pm SEM. A $P$ value $<0.05$ was considered significant.

\section{Preparation of Cortical Neurons}

Cortices from E17 Sprague-Dawley rat embryos were dissected in $0.1 \mathrm{mM}$ HEPES (Thermo Fisher Scientific, cat. \# 15630-080) supplemented Hank's balanced salt solution (HBSS, Thermo Fisher Scientific, cat. \# 14025076), HEPES-HBSS. The dissected cortices were trypsinized in $0.25 \%$ trypsin, $0.1 \mathrm{mM}$ HEPES-HBSS for $15 \mathrm{~min}$ at $37^{\circ} \mathrm{C}$; the tissue was then washed 3 times in fresh HEPES-HBSS. Afterwards, the cortices were triturated to dissociate the cells. The neurons were then plated in poly-D-lysine and laminin pre-treated $22 \times 22 \mathrm{~mm}$ coverslips (Thermo Fisher Scientific, cat. \# 12-541B) in 6 well plates (VWR International,

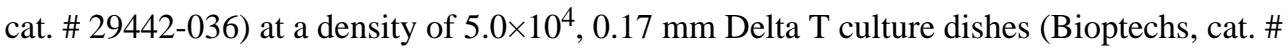
$04200417 \mathrm{C}$ ) at a density of $3.5 \times 10^{4}, 100 \times 20 \mathrm{~mm}$ tissue culture dishes (VWR, cat. \# 25382-166) at a density of $7.5 \times 10^{5}$, and 96 well plates (Sigma, cat. \# CLS3610) at a density of $2.5 \times 10^{3}$ cells per well in supplemented Neurobasal medium (NBM, Thermo Fisher Scientific, cat. \# 21103-049). The NBM was supplemented with $10 \mathrm{~mL} / \mathrm{L}$ Glutamax (Thermo Fisher Scientific, cat. \# 35050-061), 1 mg/L gentamicin solution (Thermo Fisher Scientific, cat. \# 15750-078), 20 mL/L B-27 supplement (Thermo Fisher Scientific, cat. \# 17504-044), and $50 \mathrm{~mL} / \mathrm{L}$ fetal bovine serum (Biowest, cat. \# S162H). Four hours after the neurons were plated, the medium was diluted 1:3 with serum-free supplemented NBM. After 7 days in vitro, the medium in the wells was diluted 1:2 with fresh supplemented NBM. Neurons were cultured 12-16 days in vitro (DIV) before transfection with CalPhos or 16-20 DIV prior to transduction with lentivirus.

\section{Immunocytochemistry of Cortical Neurons}

Cells were fixed with 4\% paraformaldehyde (Electron Microscopy Sciences, cat. \# 15714) for 5 minutes, and then subjected to three 5 minute washes with PBS. Prior to staining with primary antibody, cells were blocked with blocking solution (1\% bovine serum albumin, 5\% normal goat serum, $0.1 \%$ Triton X-100 in PBS) for 30 minutes. After blocking, primary antibody was diluted in blocking solution and added to cells for 1 hour. This was followed by three 5 minute washes of cells in PBS. Secondary antibody was diluted in blocking solution and added to cells for 30 minutes in the dark. After staining, cells were washed for 5 minutes three times with PBS and then mounted onto $75 \times 25 \mathrm{~mm}$ glass microscope slides (VWR, cat. \# 48300-025) in Fluoromount-G (Electron Microscopy Sciences, cat. \# 17984-25) or Fluoromount-G with DAPI. All steps were performed at room temperature. Primary antibody concentrations were as follows: chicken anti-GFP (Aves, cat. \# GFP-1020), 1:4000, mouse anti-Gephyrin (Synaptic Systems, cat. \# 147 008), 1:300, rabbit 
anti-tRFP (Evrogen, cat. \# AB234), 1:5000, mouse anti-GAD-65 (Synaptic Systems, cat. \# 198 111), 1:500, mouse anti-PSD-95 (NeuroMab, cat. \# 75-028), 1:1000, and rabbit anti$\mathrm{GABA}_{\mathrm{A}}$ receptor alpha1 (EMD Millipore, cat. \# 06-868), 1:1000. Secondary antibodies used were: goat anti-chicken Alexa Fluor 488, goat anti-mouse Alexa Fluor 594, goat anti-rabbit Alexa Fluor 594, goat anti-rabbit Alexa Fluor 647, goat anti-mouse IgG2a Alexa Fluor 594, goat anti-mouse IgG3 Alexa Fluor 594, goat anti-mouse IgG1 Alexa Fluor 594 and goat anti-mouse IgG1 Alexa Fluor 647 (Thermo Fisher Scientific, cat. \# A-11039, A-11005, R-37117, A-27040, A-21135, A-21155, A-21125, A-21240), all at dilutions of 1:1000.

\section{Image Capture and Analysis of Cortical Neurons}

Imaging of fixed, immunostained cells was done on a Bio-Rad MRC-1024 confocal microscope. Each cell was imaged as a single optical section with either an $\times 20$ objective at $1.0 \times$ zoom or an $\times 40$ objective at $2.0 \times$ zoom, unless otherwise noted. Stained levels of Gephyrin, PSD95 and $\mathrm{GABA}_{\mathrm{A}} \mathrm{R}$ were determined by measuring mean pixel intensity on dendrites from neurons with similar morphology that expressed GFE3, RandE3 or GPHN.FingR. Image analyses were performed blinded with ImageJ software. Data are represented as mean \pm SEM and were compared using a Kruskal-Wallis or Mann-Whitney test. A $P$ value $<0.05$ was considered significant. Living cells on Delta $\mathrm{T}$ culture dishes were imaged in HBSS buffer, supplemented with $10 \mathrm{mM}$ HEPES and pre-warmed to $37^{\circ} \mathrm{C}$, with an $\times 20$ air objective or $\times 60$ water objective at $1.0 \times$ zoom on an Olympus X81 inverted microscope equipped with the Delta T system (Bioptechs, cat. \# 0420-4), an EM-CCD digital camera (Hamamatsu, cat. \# C9100-02), DAPI, GFP/mCherry and Cy5.5 filter cubes (Chroma Technology, cat. \# 49000, cat. \# 59022 and \# SP105), an MS-2000 XYZ automated stage (Applied Scientific Instrumentation), an X-cite exacte mercury lamp (Excelitas Technologies) and Metamorph software (Molecular Devices). Imaging of GFP and TagRFP was done at $25 \%$ intensity with a $400 \mathrm{~ms}$ exposure to minimize bleaching and phototoxicity affects. Intensity values of puncta labeled by transcriptionally controlled GPHN.FingR-GFP in images taken at different time points were determined with ImageJ software. All analyses of cells were performed blinded. Only neurons with healthy morphology were selected for quantification, and none of the images used contained saturated pixels in the axon or dendrites of cells. Data are represented as mean pixel intensities \pm SEM and were compared using the Mann-Whitney test. A $P$ value $<0.05$ was considered significant.

\section{Cell Viability, Cytotoxicity, ATP Levels and Proteasome Activity}

$2.5 \times 10^{3}$ rat cortical neurons/well in 96 well white-walled plates were transduced with $9 \times 10^{3}$ IFUs of lentivirus with GFE3, RandE3 or GPHN.FingR for 16 hours. Cell viability and cytotoxicity were assessed using the MultiTox-Glo Multiplex Cytotoxicity Assay kit (Promega, cat. \# G9270) that measures: 1) fluorescence generated in living cells from selective uptake of a protease substrate conjugated to a fluorophore (AFC) that fluoresces on proteolytic cleavage, and 2) light produced by Luciferase from a substrate (aminoluciferin) that is activated by proteases released into the medium from dead cells. The ATP levels of neurons were measured using the CellTiter-Glo Luminescent Cell Viability Assay kit (Promega, cat. \# G7570), which uses ATP released from lysed cells and Luciferase to generate light. As a positive control for cell viability and cytotoxicity assays, the mitochondrial toxin 3-nitropropionic acid (3-NP, Sigma, cat. \# N5636) was applied to cells 
at a final concentration of $15 \mathrm{mM}$ for 48 hours $^{39}$. Proteasome activity in transduced cells was determined using the Proteasome-Glo 3-Substrate Cell-Based Assay kit (Promega, cat. \#G1180), which uses proteasome caspase-, trypsin- and chymotrypsin-like activity to produce light via aminoluciferin-based substrates and Luciferase. As a positive control for proteasome activity, the highly specific proteasome inhibitor clasto-Lactacystin-[beta]lactone was added to the medium of cells at a final concentration of $10 \mu \mathrm{M}$ for 3 hours before obtaining measurements. All assays were performed according to the instructions provided by the manufacturer. Fluorometric and luminometric measurements were taken with a Fluoroskan Ascent FL (Thermo Fisher Scientific) microplate reader. 24 wells were measured per condition. Data are represented as measurements of transduced (or positive control) cells divided by the mean of non-transduced (or vehicle control) cells \pm SEM and were compared using the Kruskal-Wallis test. A $P$ value $<0.05$ was considered significant.

\section{Preparation and Transfection of Hippocampal Neurons}

Dissociated hippocampal neurons were prepared as described previously ${ }^{40}$. Briefly, dissected hippocampi from P1-P3 Sprague Dawley rats of both sexes were dissociated enzymatically (papain, $12 \mathrm{U} / \mathrm{mL}$; Worthington) and mechanically (trituration with a Pasteur pipette). After dissociation, the cells were washed, centrifuged and plated on poly-D-lysinecoated Aclar (12 mm) coverslips at a density of 350-450 cells $/ \mathrm{mm}^{2}$. Growth medium consisted of NBM and B27 (50:1), supplemented with penicillin/streptomycin $(50 \mathrm{U} / \mathrm{mL} ; 50$ $\mu \mathrm{g} / \mathrm{mL})$ and $0.5 \mathrm{mM}$ Glutamax. Fetal bovine serum (2\%, Hyclone, GE Healthcare, cat. \# SH30088.02) was added at the time of plating. After 5 days, half the medium was changed to a medium without serum, but with Ara-C added ( $5 \mu \mathrm{M}$; Sigma-Aldrich; cat. \# C1768) to limit proliferation of non-neuronal cells. Twice a week, half of the growth medium was replaced with serum and Ara-C free medium. Neurons were transfected at 11-13 DIV using Lipofectamine 2000, as previously described ${ }^{40}$. To avoid synaptic scaling after gephyrin ablation, cultures were treated with TTX $(1 \mu \mathrm{M}) 24 \mathrm{~h}$ before recording. By that time, expression of FingR constructs was observed

\section{Electrophysiology of Hippocampal Neurons}

Coverslips containing transfected neurons were transferred to a recording chamber and perfused with a recording solution which consisted of HBSS containing: $1.2 \mathrm{mM} \mathrm{CaCl}_{2}$, and $1.0 \mathrm{mM} \mathrm{MgCl} 2,10 \mathrm{mM}$ Hepes, $2.0 \mathrm{mM}$ glucose, $\mathrm{pH}$ 7.3. The osmolarity of the solution was adjusted to $290 \mathrm{mOsm} / \mathrm{kg}$. Whole-cell voltage clamp recordings were obtained with borosilicate glass pipettes (4-6 M $\Omega$ ). For mIPSC recordings, pipettes were filled with an internal solution containing: $100 \mathrm{mM} \mathrm{CsMeSO} 3,20 \mathrm{mM} \mathrm{CsCl}, 2.5 \mathrm{MgCl}_{2}, 70 \mathrm{mM}$ Hepes, $0.6 \mathrm{mM}$ Cs-EGTA, $4 \mathrm{mM}$ Mg-ATP, $0.4 \mathrm{mM}$ Tris-GTP, pH 7.3 and clamped at $0 \mathrm{mV}$ in the presence of TTX $(1 \mu \mathrm{M})$. Evoked GABA currents were recorded with an internal solution containing: $120 \mathrm{mM} \mathrm{CsCl}, 2.0 \mathrm{mM} \mathrm{MgCl} 2,1.0 \mathrm{mM} \mathrm{CaCl}_{2}, 10 \mathrm{mM}$ Hepes, $11 \mathrm{mM}$ CsEGTA, $4 \mathrm{mM}$ Mg-ATP, $1 \mathrm{mM}$ TEA, pH 7.3. After a stable baseline, a high concentration of GABA $(1 \mathrm{mM})$ was added to the bath during 1 minute and then washed out. For mEPSC recordings pipettes were filled with an internal solution containing: $100 \mathrm{mM} \mathrm{CsMeSO} 3,20$ $\mathrm{mM} \mathrm{CsCl}, 2.5 \mathrm{MgCl} 2,70 \mathrm{mM}$ Hepes, $0.6 \mathrm{mM}$ Cs-EGTA, 4 mM Mg-ATP, $0.4 \mathrm{mM}$ TrisGTP, $\mathrm{pH}$ 7.3. Miniature excitatory and inhibitory post-synaptic currents (mEPSCs and 
mIPSCS) were recorded at holding potentials of $-70 \mathrm{mV}$ and $0 \mathrm{mV}$, respectively, in the presence of TTX $(1 \mu \mathrm{M})$.

All recordings were performed at room temperature. Signals were low-pass filtered at $2 \mathrm{kHz}$ and digitized at $10 \mathrm{kHz}$ using a Multiclamp 700B amplifier, Digidata 1440 digitizer and pClamp 10 software (Molecular Devices). Comparisons of parameters for mIPSCs and mEPSCs were made using a Kruskal-Wallis test followed by a Dunn's multiple parameter comparison. Responses to GABA applications were analyzed using 2-tailed unpaired MannWhitney test. A $P$ value $<0.05$ was considered significant. All data are expressed as mean \pm SEM.

\section{Noise analysis}

To see if unclustering of $\mathrm{GABA}_{\mathrm{A}}$ receptors affects their kinetic properties, we performed stationary noise analysis of $\mathrm{GABA}_{\mathrm{A}}$ receptors activated by the exogenous application of GABA $(1 \mathrm{mM})$ as previously described ${ }^{41}$. Briefly, a region of $50 \mathrm{~s}$ after peak GABA activation was high pass filtered at $1 \mathrm{~Hz}$ and a power spectrum was derived. This spectrum was fitted with the sum of three Laurentzian functions of the form:

$$
L(f)=\sum_{i=1}^{n} S_{i} /\left(1+\left(f / f_{c i}\right)^{2}\right)
$$

where $L(f)$ is the spectral density at frequency $f . S_{i}$ is the power of the spectrum at $f=0$ and $f_{c i}$ is the cutoff frequency at which the spectral power is half. The corresponding time constants $\left(\tau_{i}\right)$ are derived by the relationship $\tau_{i}=1 /\left(2 \pi f c_{i}\right) 41$.

\section{Zebrafish Husbandry and Experimentation}

Wild-type $\mathrm{AB}$ and $\mathrm{AB} / \mathrm{TL}$ zebrafish (Danio rerio) were raised and nucleic acid injections were performed as described previously ${ }^{42}$. Embryos were maintained at $28^{\circ} \mathrm{C}$ for up to 5 days post fertilization (dpf) in egg water $\left(1.19 \mathrm{~g} \mathrm{NaCl}, 0.377 \mathrm{~g} \mathrm{CaSO}_{4} \cdot 2 \mathrm{H}_{2} \mathrm{O}, 265 \mu \mathrm{L}\right.$ methylene blue, filled to $5 \mathrm{~L}$ with $\mathrm{ddH}_{2} \mathrm{O}$ ). Within the $28^{\circ} \mathrm{C}$ incubator, the fish were exposed to a 13/11 hour day/night light cycle. At the zygote stage, zebrafish were left uninjected (control) or were injected with 45-70 pg of DNA, along with $35 \mathrm{pg}$ of Tol2 Transposase ${ }^{43}$ mRNA27 $\mathrm{S}$ to facilitate mosaic integration of the $\mathrm{pMT}(3 \mathrm{mnx} 1)$ plasmid DNA into the zebrafish genome. Before behavior experiments (i.e. spontaneous tail flick), 17-22 hour post fertilization zebrafish embryos in egg water were carefully dechorionated with a pair of \#5 forceps (Fine Science Tools). $200 \mu \mathrm{L}$ of egg water supplemented with 0.003\% 1-phenyl 2thiourea (PTU, Sigma-Aldrich, cat. \# P7629) - to inhibit pigment formation - was added to individual wells of a 96-well plate, and individual zebrafish were placed into each of the filled wells with a glass pipette. Gender could not be determined due to the age of the fish ${ }^{44}$. Zebrafish tail flicking behavior was recorded (40 frames per second) from below the well plate using a compact USB 3.0 CMOS camera (Cat \#DCC1545M, Thorlabs). After tail flick imaging, zebrafish were kept overnight at $28^{\circ} \mathrm{C}$ within the 96 -well plate. At $2 \mathrm{dpf}$, zebrafish injected with the spinal cord neuron expressing GFE construct were anesthetized with $0.015-0.03 \%$ MS-222 (Argent Laboratories), embedded in 1-1.5\% SeaPlaque agarose within a 30X Danieau's solution (17.4 mM NaCl, $0.21 \mathrm{mM} \mathrm{KCl}, 0.12 \mathrm{mM} \mathrm{MgSO}_{4}, 0.18$ 
$\mathrm{mM} \mathrm{Ca}\left(\mathrm{NO}_{3}\right)_{2}, 1.5 \mathrm{mM}$ HEPES$)$, and imaged using a Plan Apochromat $\times 20$ objective on a commercial LSM 700 confocal microscope (Carl Zeiss AG), as similarly described ${ }^{45}$. Mosaic-labeled GFP positive cells were visualized throughout the zebrafish spinal cord using the tiled confocal stack feature of the microscope. For immunostaining of zebrafish, 1-2 dpf zebrafish were anesthetized in 0.03\% MS-222 and were fixed in 2\% Trichoroacetic acid for 3 hours at room temperature. After 5 washes in PBS pH 7.4 with $0.5 \%$ Triton X-100 (PBT), fish were blocked overnight in blocking buffer (PBS pH 7.4, $1 \%$ bovine serum albumin, $1 \%$ DMSO, $0.1 \%$ Triton $\mathrm{X}-100,2 \%$ normal goat serum) at $4{ }^{\circ} \mathrm{C}$ on an orbital shaker. Mouse anti-Gephyrin antibody (Synaptic Systems, cat. \# 147 111) was applied at a 1:250 dilution in blocking buffer and the embryos were left at $4{ }^{\circ} \mathrm{C}$ on an orbital shaker for $72-96$ hours. After 5 washes in PBT, goat anti-mouse Alexa Fluor 594 antibody (Thermo Fisher Scientific, cat. \#A-11005) was applied at a 1:2000 dilution in blocking buffer, and the sample was left on an orbital shaker at $4{ }^{\circ} \mathrm{C}$ for $14-18$ hours. Following 5 washes in PBT, samples were washed in PBS pH 7.4. For clearing, samples were progressively dehydrated in glycerol, ultimately ending in a solution of $75 \%$ glycerol / 25\% PBS pH 7.4. Samples were placed on \#1.5 coverslips and were imaged with a commercial LSM 780 confocal microscope (Carl Zeiss AG) using an $\times 63$ water objective. Analyses of Gephyrin levels in confocal images were performed blinded with ImageJ software. All data are expressed as mean \pm SEM. Zebrafish were included in analysis that had at least 20 labeled motoneurons. No randomization was used.

\section{Stereotaxic Intracranial Injections}

To examine inhibitory synaptic inputs, a total of $1 \mu$ AAV-DIO-GFE3-GFP ('cre-on') mixed at a 2:1 ratio with AAV-FAS-ChR2-mCherry ('cre-off' ${ }^{37}$ ) was delivered by intracranial injection into the striatum of Adora2a-cre mice (GENSAT: B6.FVB $(\mathrm{Cg})$ $\mathrm{Tg}$ (Adora2acre)KG139Gsat/Mmucd) (P18-P22) of both sexes maintained under deep isoflurane anesthesia. The non-ablating gephyrin intrabody (AAV-DIO-GPHN.FingR$\mathrm{GFP}^{11}$ ) was used as an internal control in the other hemisphere of each mouse. Note that AAV-EF1a-DIO-GFE3 and AAV-EF1a-DIO-GPHN.FingR are AAV serotype 9, AAV-EF1aFAS-ChR2-mCherry is serotype 8 and $3.8 \times 10^{10}$ genome copies (GC) at a titer of $3.8 \times 10^{13}$ $\mathrm{GC} / \mathrm{mL}$ were used. To test excitatory inputs, AAV-DIO-GFE3-GFP or AAV-DIOGPHN.FingR-GFP mixed with AAV-hSyn-cre at a 100:1 GC-ratio was injected into the striatum of age-matched wildtype mice of both sexes (C57B1/6, Charles River). Injection coordinates were $0.7 \mathrm{~mm}$ anterior from Bregma, $1.8 \mathrm{~mm}$ lateral, and $2.45 \mathrm{~mm}$ below pia, and expression time was at 12-14 days.

\section{Slice Preparation and Electrophysiology}

Acute sagittal brain slices were prepared, and electrophysiological recordings obtained, as described before ${ }^{46}$, with the following variations: Spiny projection neurons of the indirect pathway were identified by GFP expression and their electrical properties, and synaptic currents were recorded using a high-chloride pipette solution containing (in $\mathrm{mM}$ ) $125 \mathrm{CsCl}$, 10 TEA-Cl, 0.1 EGTA (CsOH), 10 HEPES, 3.3 QX-314 (Cl-), $1.8 \mathrm{MgCl}_{2}, 4 \mathrm{Na}_{2}$-ATP, 0.3 Na-GTP, $8 \mathrm{Na}_{2}$-phosphocreatine ( $\mathrm{pH} 7.3$ adjusted with $\mathrm{CsOH} ; 295 \mathrm{mOsm} \cdot \mathrm{kg}^{-1}$ ). GABA receptor-mediated currents were recorded as inward currents in voltage-clamp mode at $\mathrm{V}_{\text {hold }}$ $=-70 \mathrm{mV}$ in the presence of the AMPA and NMDA-type glutamate receptor inhibitors 
NBQX and (R)-CPP (10 $\mu \mathrm{m}$ each). Excitatory currents were isolated by adding SR95331 $(10 \mu \mathrm{M})$ to the bath solution. Tonic $\mathrm{GABA}_{\mathrm{A}}$ receptor mediated currents were defined as the difference in holding current before and after bath application of SR95331 $(10 \mu \mathrm{M})$. Pipette resistance was $2.5-3 \mathrm{M} \Omega$, and all recordings were performed at $33-34^{\circ} \mathrm{C}$. Errors due to the voltage drop across the series resistance $(<15 \mathrm{M} \Omega)$ were left uncompensated. ChR2 was activated by a single $1 \mathrm{~ms}$ pulse of light delivered by a $473 \mathrm{~nm}$ laser using full-field illumination through the objective $\left(\sim 3 \mathrm{~mW} \cdot \mathrm{mm}^{-2}\right)$ at $30 \mathrm{~s}$ intervals. All pharmacological reagents were obtained from Tocris.

\section{Data Acquisition and Analysis}

Membrane currents were acquired as described previously ${ }^{46}$, and analyzed in Igor Pro (Wavemetrics). For display and analysis of optically evoked synaptic membrane currents, 10 consecutive traces were averaged for each cell. Data are represented as mean \pm SEM (bars) overlaid over individual data points (gray circles) and were compared using the nonparametric Mann-Whitney test. $P$ values are reported directly, and values smaller than 0.05 were considered statistically significant.

\section{Statistics}

No statistical methods were used to predetermine sample size. Nonparametric tests were used throughout, as the data are not normally distributed. Data are represented as mean \pm SEM. Statistical comparisons are between groups with similar variances.

\section{Supplementary Material}

Refer to Web version on PubMed Central for supplementary material.

\section{Acknowledgments}

We thank M. Roth for technical support, Y. Davis and C. Tian for assistance in analyzing the data, L. Chen for use of his microplate reader, and E. Liman for helpful suggestions on the manuscript. Lentivirus was created by $\mathrm{L}$. Asatryan at USC School of Pharmacy Lentiviral Core Laboratory. This work was supported by NIH (grants AI085583 and GM083898 to R.W.R., NS046579 to B.L.S. and NS081687 to D.B.A.), by the Human Frontier Science Program (to Y.D.K and D.B.A), by the Canadian Institutes of Health Research (MOP12942 to Y.D.K. and MOP286161 to P.D.K) and by the McKnight foundation (to D.B.A).

\section{References}

1. Cabot JB, Bushnell A, Alessi V, Mendell NR. Postsynaptic gephyrin immunoreactivity exhibits a nearly one-to-one correspondence with gamma-aminobutyric acid-like immunogold-labeled synaptic inputs to sympathetic preganglionic neurons. J Comp Neurol. 1995; 356:418-432. [PubMed: 7642803]

2. Craig AM, Banker G, Chang W, McGrath ME, Serpinskaya AS. Clustering of gephyrin at GABAergic but not glutamatergic synapses in cultured rat hippocampal neurons. J Neurosci. 1996; 16:3166-3177. [PubMed: 8627355]

3. Feng G, et al. Dual requirement for gephyrin in glycine receptor clustering and molybdoenzyme activity. Science. 1998; 282:1321-1324. [PubMed: 9812897]

4. Capecchi MR. Altering the genome by homologous recombination. Science. 1989; 244:1288-1292. [PubMed: 2660260]

5. McManus MT, Sharp PA. Gene silencing in mammals by small interfering RNAs. Nature reviews. Genetics. 2002; 3:737-747. 
6. Hedstrom KL, Ogawa Y, Rasband MN. AnkyrinG is required for maintenance of the axon initial segment and neuronal polarity. J Cell Biol. 2008; 183(4):635-640. [PubMed: 19001126]

7. Incontro S, Asensio CS, Edwards RH, Nicoll RA. Efficient, complete deletion of synaptic proteins using CRISPR. Neuron. 2014; 83:1051-1057. [PubMed: 25155957]

8. Deshaies RJ, Joazeiro CA. RING domain E3 ubiquitin ligases. Annu Rev Biochem. 2009; 78:399_ 434. [PubMed: 19489725]

9. Caussinus E, Kanca O, Affolter M. Fluorescent fusion protein knockout mediated by anti-GFP nanobody. Nat Struct Mol Biol. 2012; 19:117-121.

10. Yeh JT, Binari R, Gocha T, Dasgupta R, Perrimon N. PAPTi: a peptide aptamer interference toolkit for perturbation of protein-protein interaction networks. Sci Rep. 2013; 3:1156. [PubMed: 23362456]

11. Gross GG, et al. Recombinant probes for visualizing endogenous synaptic proteins in living neurons. Neuron. 2013; 78:971-985. [PubMed: 23791193]

12. Galban S, Duckett CS. XIAP as a ubiquitin ligase in cellular signaling. Cell Death Differ. 2010; 17:54-60. [PubMed: 19590513]

13. Kins S, Betz H, Kirsch J. Collybistin, a newly identified brain-specific GEF, induces submembrane clustering of gephyrin. Nat Neurosci. 2000; 3:22-29. [PubMed: 10607391]

14. Chen L, Wang H, Vicini S, Olsen RW. The gamma-aminobutyric acid type A (GABAA) receptorassociated protein (GABARAP) promotes GABAA receptor clustering and modulates the channel kinetics. Proc Natl Acad Sci U S A. 2000; 97:11557-11562. [PubMed: 10984509]

15. Jacob TC, et al. Gephyrin regulates the cell surface dynamics of synaptic GABAA receptors. J Neurosci. 2005; 25:10469-10478. [PubMed: 16280585]

16. Wilson CJ. GABAergic inhibition in the neostriatum. Prog Brain Res. 2007; 160:91-110. [PubMed: 17499110]

17. Ade KK, Janssen MJ, Ortinski PI, Vicini S. Differential tonic GABA conductances in striatal medium spiny neurons. J Neurosci. 2008; 28:1185-1197. [PubMed: 18234896]

18. Santhakumar V, Jones RT, Mody I. Developmental regulation and neuroprotective effects of striatal tonic GABAA currents. Neuroscience. 2010; 167:644-655. [PubMed: 20206233]

19. Tritsch NX, Oh WJ, Gu C, Sabatini BL. Midbrain dopamine neurons sustain inhibitory transmission using plasma membrane uptake of GABA, not synthesis. eLife. 2014; 3:e01936. [PubMed: 24843012]

20. Zelenchuk TA, Bruses JL. In vivo labeling of zebrafish motor neurons using an mnx1 enhancer and Gal4/UAS. Genesis. 2011; 49:546-554. [PubMed: 21538811]

21. Hirata H, Takahashi M, Yamada K, Ogino K. The biological role of the glycinergic synapse in early zebrafish motility. Neuroscience research. 2011; 71:1-11. [PubMed: 21712054]

22. Cui WW, et al. The zebrafish shocked gene encodes a glycine transporter and is essential for the function of early neural circuits in the CNS. J Neurosci. 2005; 25:6610-6620. [PubMed: 16014722]

23. Hirata $\mathrm{H}$, et al. Zebrafish bandoneon mutants display behavioral defects due to a mutation in the glycine receptor beta-subunit. Proc Natl Acad Sci U S A. 2005; 102:8345-8350. [PubMed: 15928085]

24. Hirata H, Carta E, Yamanaka I, Harvey RJ, Kuwada JY. Defective glycinergic synaptic transmission in zebrafish motility mutants. Frontiers in molecular neuroscience. 2009; 2:26. [PubMed: 20161699]

25. Ganser LR, et al. Distinct phenotypes in zebrafish models of human startle disease. Neurobiol Dis. 2013; 60:139-151. [PubMed: 24029548]

26. Yu W, et al. Gephyrin clustering is required for the stability of GABAergic synapses. Mol Cell Neurosci. 2007; 36:484-500. [PubMed: 17916433]

27. Mora RJ, Roberts RW, Arnold DB. Recombinant probes reveal dynamic localization of CaMKIIalpha within somata of cortical neurons. J Neurosci. 2013; 33:14579-14590. [PubMed: 24005308]

28. Banaszynski LA, Wandless TJ. Conditional control of protein function. Chem Biol. 2006; 13:1121. [PubMed: 16426967] 
29. Sakamoto KM, et al. Protacs: chimeric molecules that target proteins to the Skp1-Cullin-F box complex for ubiquitination and degradation. Proc Natl Acad Sci U S A. 2001; 98:8554-8559. [PubMed: 11438690]

30. Varley ZK, et al. Gephyrin regulates GABAergic and glutamatergic synaptic transmission in hippocampal cell cultures. J Biol Chem. 2011; 286:20942-20951. [PubMed: 21507951]

31. Fan X, Jin WY, Lu J, Wang J, Wang YT. Rapid and reversible knockdown of endogenous proteins by peptide-directed lysosomal degradation. Nat Neurosci. 2014; 17:471-480. [PubMed: 24464042]

32. Dev KK. PDZ domain protein-protein interactions: a case study with PICK1. Curr Top Med Chem. 2007; 7:3-20. [PubMed: 17266593]

33. Young Kim H, Young Yum S, Jang G, Ahn D-R. Discovery of a non-cationic cell penetrating peptide derived from membrane-interacting human proteins and its potential as a protein delivery carrier. Scientific Reports. 2015; 5:11719. [PubMed: 26114640]

34. Zhang F, et al. Multimodal fast optical interrogation of neural circuitry. Nature. 2007; 446:633639. [PubMed: 17410168]

35. Hayashi-Takagi A, et al. Labelling and optical erasure of synaptic memory traces in the motor cortex. Nature. 2015; 525:333-338. [PubMed: 26352471]

\section{Methods-only References}

36. Olson CA, Roberts RW. Design, expression, and stability of a diverse protein library based on the tenth fibronectin type III domain. Protein Science. 2007; 16:476-484. [PubMed: 17322532]

37. Saunders A, Johnson CA, Sabatini BL. Novel recombinant adeno-associated viruses for Cre activated and inactivated transgene expression in neurons. Frontiers in neural circuits. 2012; 6:47. [PubMed: 22866029]

38. Blobel G, Sabatini DD. Controlled Proteolysis of Nascent Polypeptides in Rat Liver Cell Fractions. Journal of Cell Biology. 1970; 45:130-145. [PubMed: 5458992]

39. Pang Z, Geddes JW. Mechanisms of cell death induced by the mitochondrial toxin 3-nitropropionic acid: acute excitotoxic necrosis and delayed apoptosis. J Neurosci. 1997; 17:3064-3073. [PubMed: 9096141]

40. Hudmon A, et al. A mechanism for $\mathrm{Ca} 2+/$ calmodulin-dependent protein kinase II clustering at synaptic and nonsynaptic sites based on self-association. J Neurosci. 2005; 25:6971-6983. [PubMed: 16049173]

41. De Koninck Y, Mody I. Noise analysis of miniature IPSCs in adult rat brain slices: properties and modulation of synaptic GABAA receptor channels. Journal of Neurophysiology. 1994; 71:1318 1335. [PubMed: 8035217]

42. Detrich, HW., Westerfield, M., Zon, LI. Essential zebrafish methods : cell and developmental biology. Elsevier: 2009.

43. Kawakami K, Shima A. Identification of the Tol2 transposase of the medaka fish Oryzias latipes that catalyzes excision of a nonautonomous Tol2 element in zebrafish Danio rerio. Gene. 1999; 240:239-244. [PubMed: 10564832]

44. Uchida D, Yamashita M, Kitano T, Iguchi T. Oocyte apoptosis during the transition from ovary-like tissue to testes during sex differentiation of juvenile zebrafish. J Exp Biol. 2002; 205:711-718. [PubMed: 11914381]

45. Dempsey WP, et al. In vivo single-cell labeling by confined primed conversion. Nat Methods. 2015; 12:645-648. [PubMed: 25984699]

46. Straub C, Tritsch NX, Hagan NA, Gu C, Sabatini BL. Multiphasic modulation of cholinergic interneurons by nigrostriatal afferents. J Neurosci. 2014; 34:8557-8569. [PubMed: 24948810] 


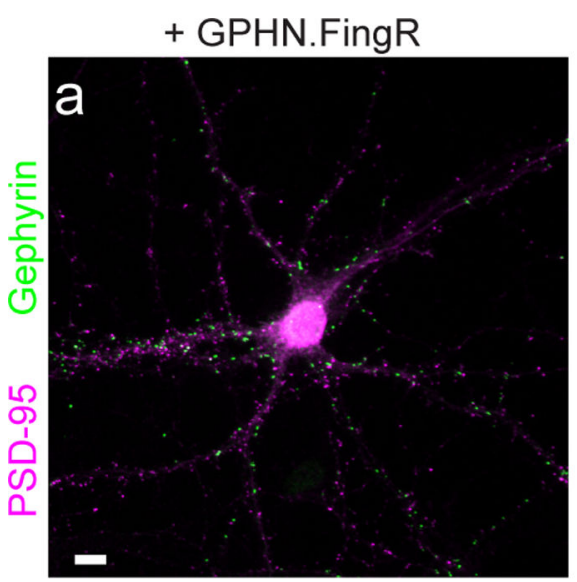

d
+ RandE3
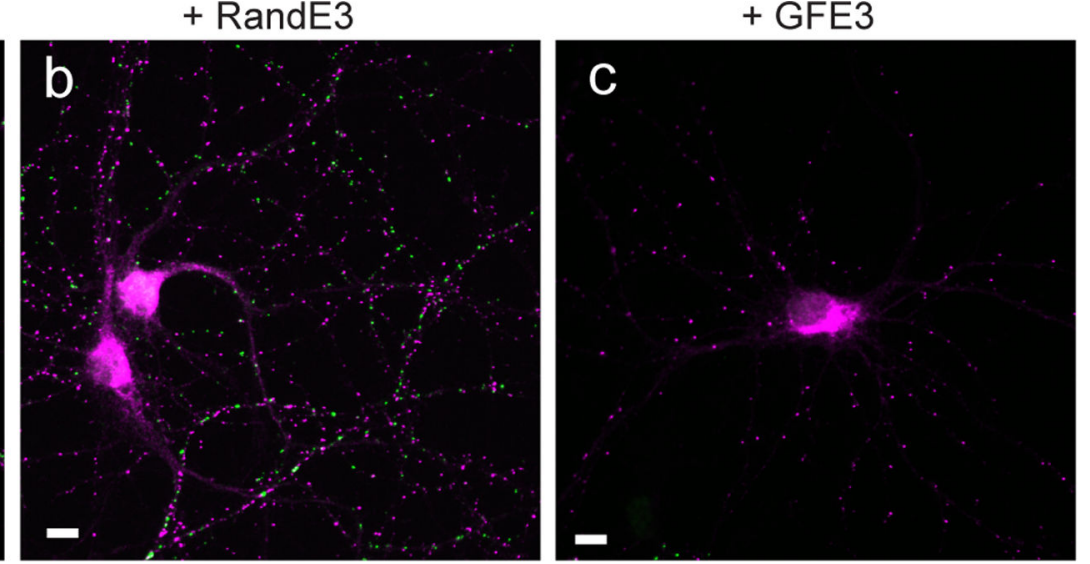

+ RandE3

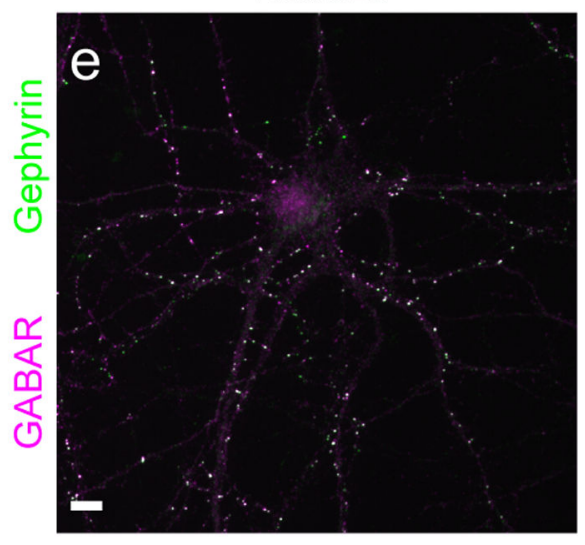

+ GFE3

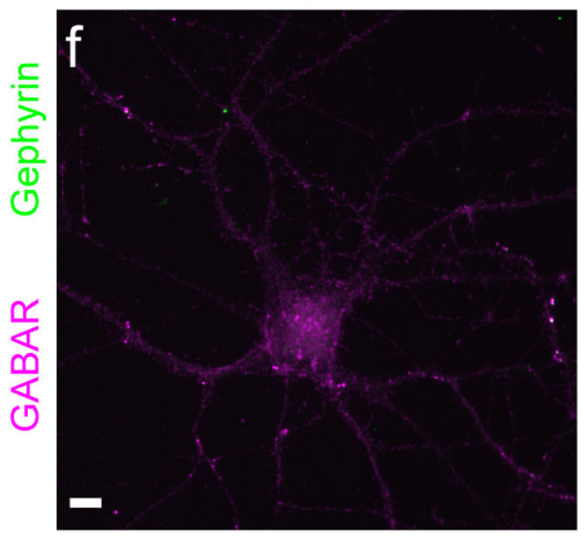

Figure 1. GFE3 specifically ablates Gephyrin

(a-c) Immunostaining showing Gephyrin (green) and PSD-95 (magenta) localization at inhibitory and excitatory synapses, respectively, after expression of GPHN.FingR (a), RandE3 (b) or GFE3 (c) in cultured rat cortical neurons. (d) Western blot showing the expression of Gephyrin, the alpha1 subunit of the GABA $_{A}$ receptor, PSD-95, the GluA1 subunit of the AMPA receptor, the GluN2B subunit of the NMDA receptor, or the Gephyrin interacting proteins Collybistin II and GABARAP in neurons not transduced (Control) or transduced with GFE3, RandE3 or GPHN.FingR. Gephyrin expression is reduced in neurons 
expressing GFE3 ( $80 \pm 3 \%, P=0.01$, Kruskal-Wallis). No significant change is observed in the steady-state levels of the other proteins ( $P>0.8$, Kruskal-Wallis; $\mathrm{n}=4$ replicate blots). $\left(\right.$ e, f) Staining for the alpha1 subunit of the $\mathrm{GABA}_{\mathrm{A}}$ receptor (magenta) and Gephyrin (green) in cortical neuron expressing RandE3 (e) or GFE3 (f). Scale bar represents $10 \mu \mathrm{m}$. 
a

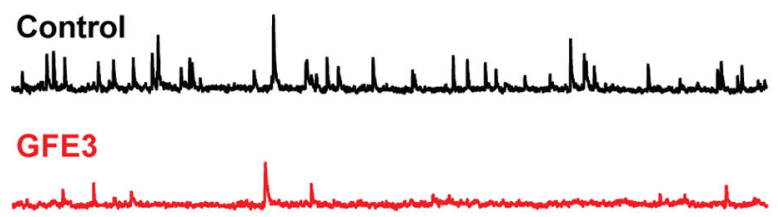

RandE3
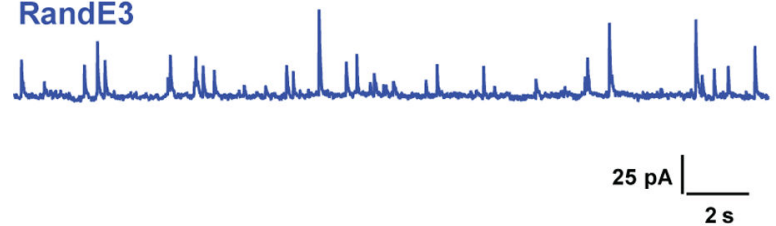

C

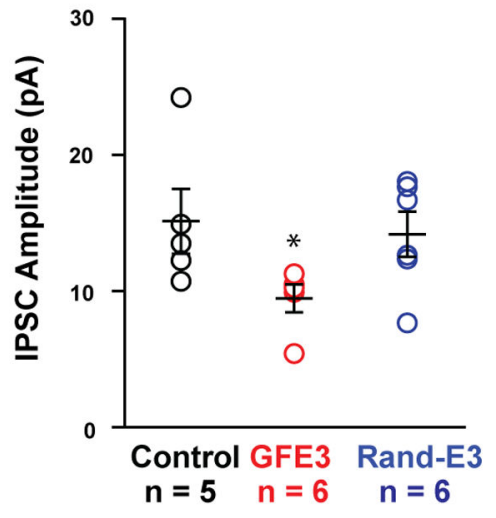

e

Control

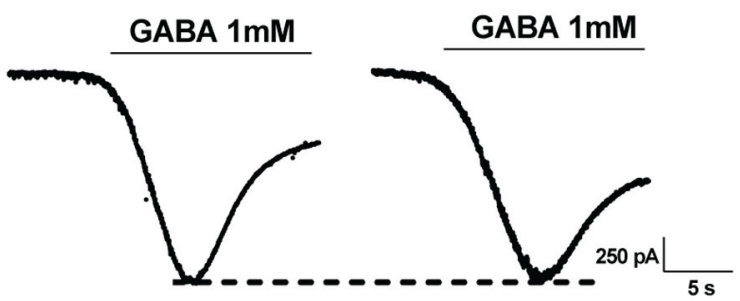

d b

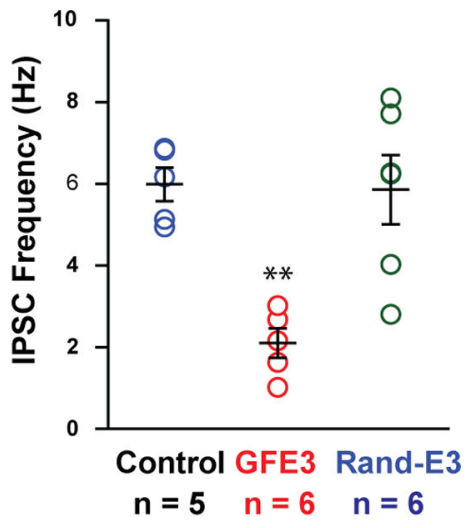

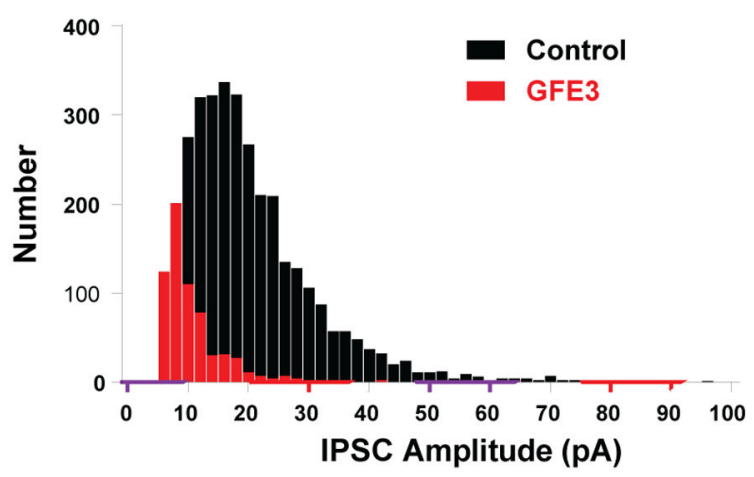

Figure 2. Gephyrin ablation reduces the amplitude and frequency of mIPSCs without affecting GABA-evoked currents

(a) Representative traces of mIPSCs obtained from dissociated rat hippocampal neurons expressing GFE3 or RandE3, or control neurons. Cells expressing GFE3 exhibit a significant reduction in frequency (b; $P<0.002$, Kruskal-Wallis) and amplitude (c; $P<0.02$, KruskalWallis) of miniature inhibitory postsynaptic currents (mIPSCs) vs. cells expressing RandE3 or control cells. (d) Histogram of the distribution of mIPSC amplitude in a control cell and in a cell expressing GFE3. $n=5$ cells for control, $n=6$ cells for GFE3 and RandE3. (e). 
Quantification of the peak amplitude of evoked GABA currents recorded in GFE3 transfected and control cells (f; $P>0.5$, Mann-Whitney). $\mathrm{n}=7$ independent experiments. Bars indicate means \pm SEM. 

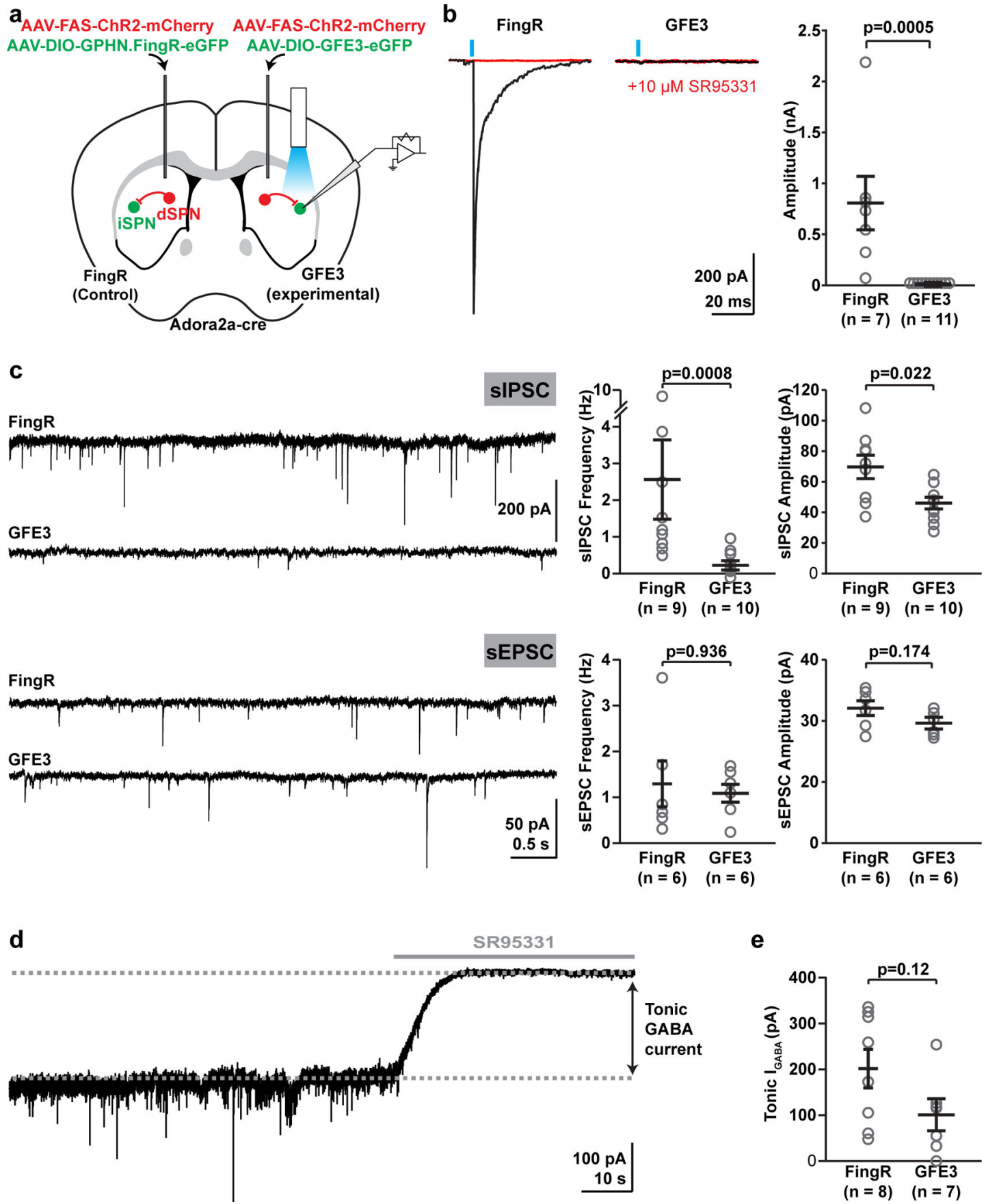

Figure 3. Loss of synaptic GABAergic currents in the intact mouse brain from expression of GFE3

(a) Schematic of experimental layout. (b) Evoked synaptic currents in control and GFE3expressing indirect pathway spiny projection neurons (iSPNs) in control conditions (black) and after slices were exposed to SR95331 (10 $\mu \mathrm{M})$, a GABAA receptor antagonist (red). (c) Spontaneous inhibitory currents (sIPSCs, top) were reduced by GFE3 compared with control in both frequency $(P<0.0008$, Mann-Whitney) and amplitude $(P<0.03$, Mann-Whitney), but no effect was observed on the frequency $(P>0.9$, Mann-Whitney) or amplitude $(P>$ 
0.17, Mann-Whitney) of excitatory currents (sEPSCs, bottom). Representative example traces are shown on the left, quantification summaries on the right. (d) Tonic GABA $A$ receptor current was quantified as the change in baseline holding current following application of SR95331 $(10 \mu \mathrm{M})$. (e) Expression of GFE3 resulted in a tendency to reduction of tonic $\mathrm{GABA}_{\mathrm{A}}$ receptor current, $(P=0.12$, Mann-Whitney). For all experiments, grey circles indicate individual cells from a total of 4 animals (inhibitory inputs) or 2 animals (excitatory inputs), bars indicate means \pm SEM. 


\section{a}
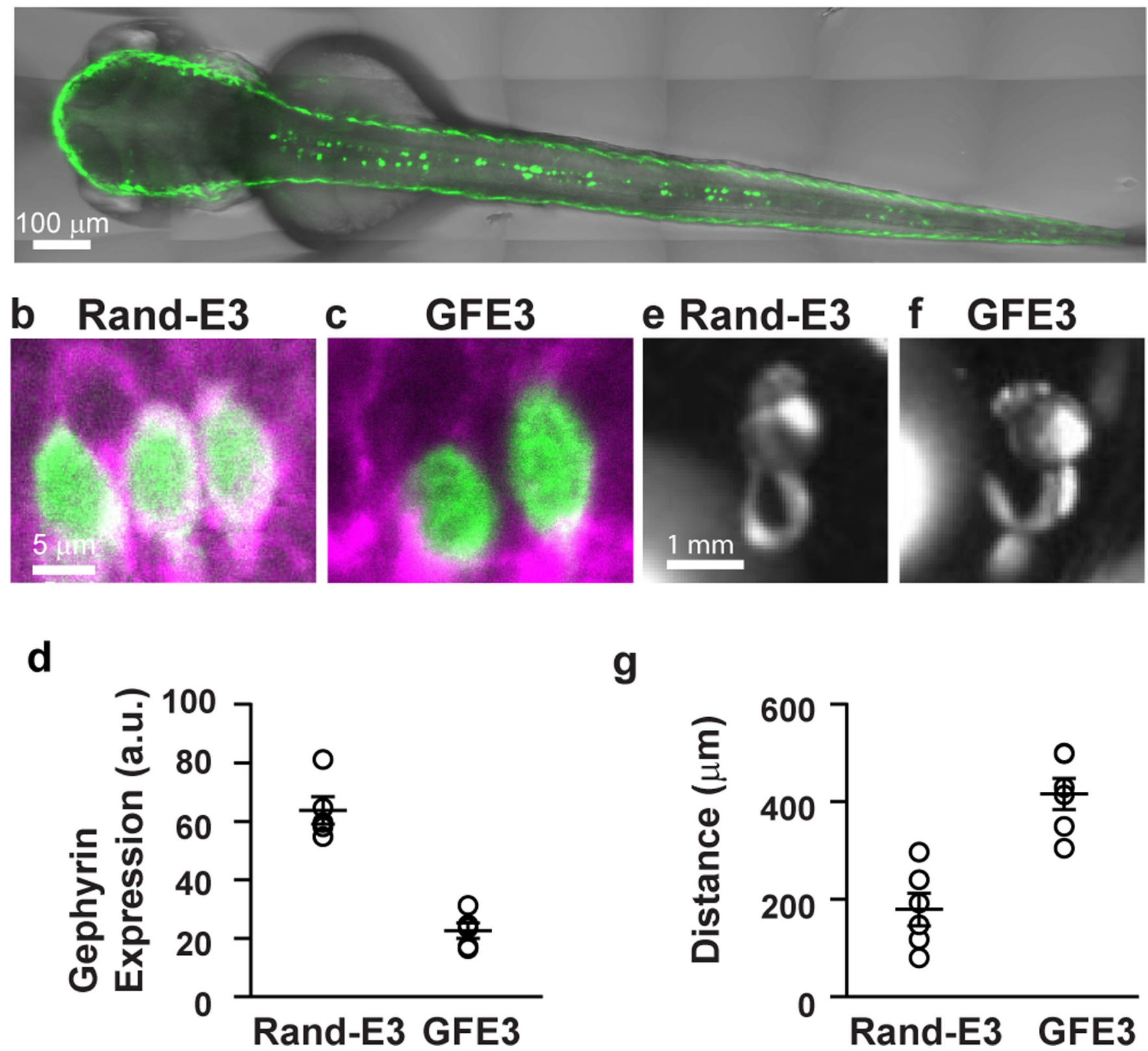

g

Figure 4. GFE3 expression in zebrafish spinal cord results in Gephyrin ablation and behavioral deficits

(a) A confocal image of a live zebrafish age 2 days post fertilization (dpf) shows mosaic expression of GFE3-GFP driven by an enhancer element of the mnx 1 gene that restricts expression to spinal motoneurons. (b,c) Immunostaining of Gephyrin (magenta) and GFP (green) in motoneurons expressing RandE3-GFP (b) or GFE3 (c). Scale bar represents $5 \mu \mathrm{m}$. (d) Gephyrin levels in cells expressing RandE3-GFP (64 \pm 5 a.u.; $\mathrm{n}=5$ fish, 6 cells per fish, 3 independent experiments) and in cells expressing GFE3-GFP (23 \pm 2 a.u.; $P<0.008$, Mann-Whitney; $\mathrm{n}=5$ fish, 6 cells per fish, 3 independent experiments). (e) Zebrafish expressing RandE3-GFP, age 24 hours post fertilization, exhibit normal spontaneous tail 
flicking, including touching tip of the tail to the base. (f) Zebrafish expressing GFE3-GFP in motoneurons are unable to curl their tails sufficiently to touch the tip to the base. (g) Quantitation of the minimum distance between the base of the tail and the tip, measured during 5 spontaneous flicks, reveals a $>100 \%$ increase in zebrafish that express GFE3-GFP vs. those expressing RandE3-GFP ( $P<0.003$, Mann-Whitney; $\mathrm{n}=5$ fish, 5 flicks per fish). Bars indicate means \pm SEM. 

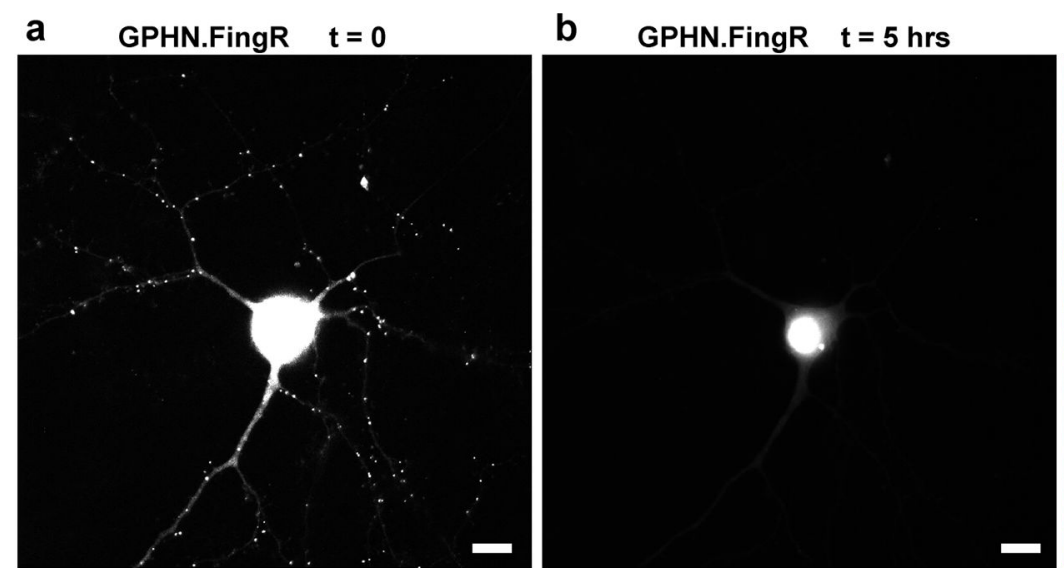

C Gephyrin GFE3 $\mathbf{t}=\mathbf{5} \mathbf{h r s}$

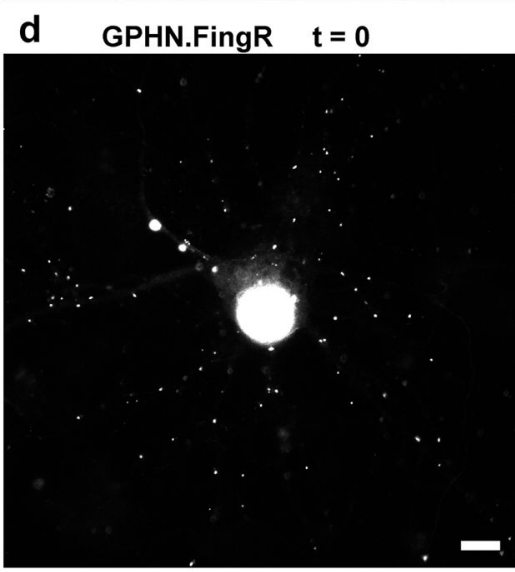

g

GPHN.Fing $\mathbf{t}=0$ GPHN.FingR $t=72 \mathrm{hr}$

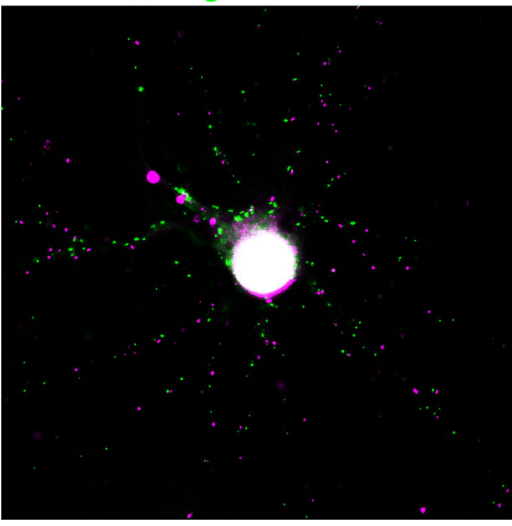

e

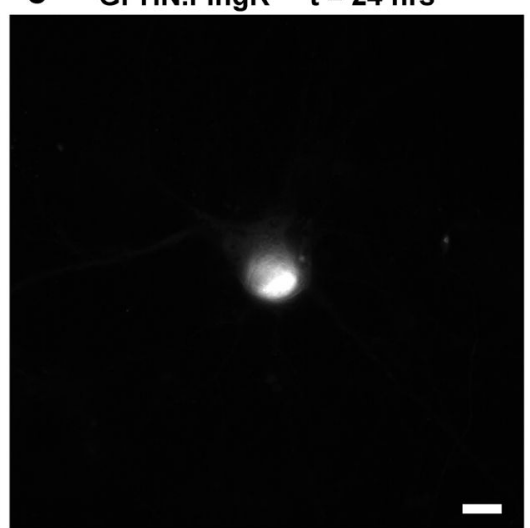

h GPHN.Fing R

GAD-65

Gephyrin

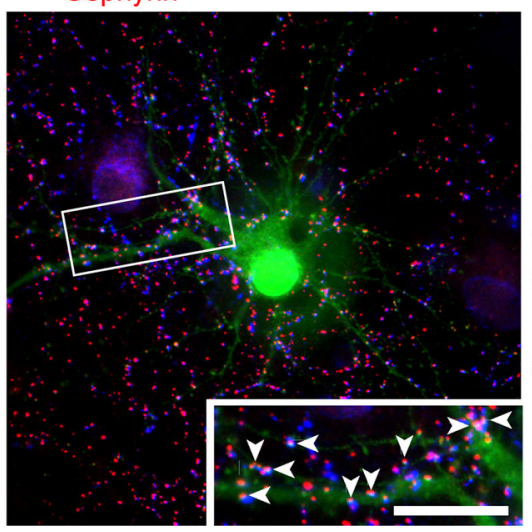

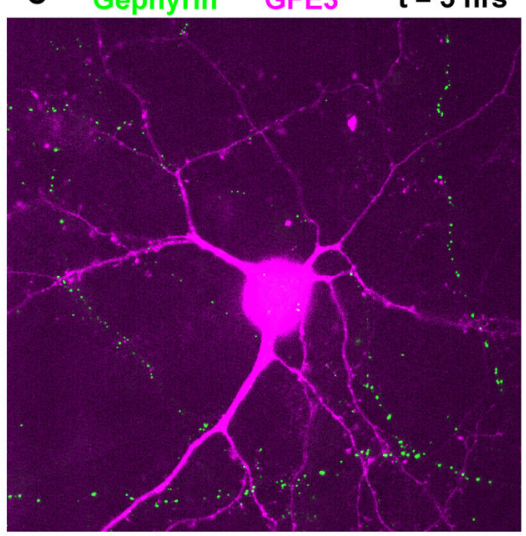

f GPHN.FingR $t=72 \mathrm{hrs}$

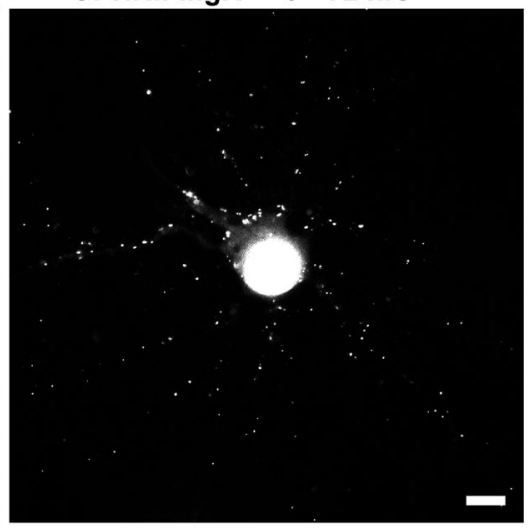

i

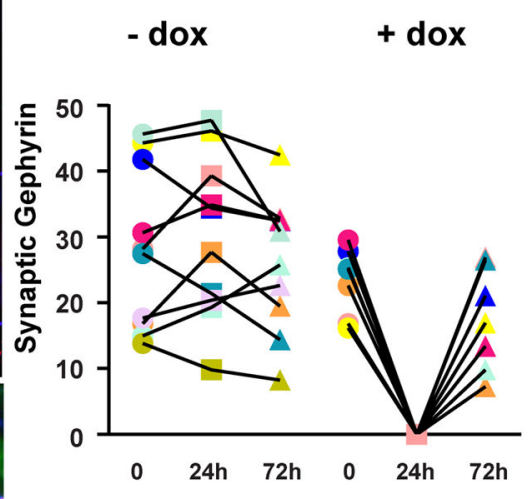

Figure 5. GFE3 mediates transient and reversible ablation of Gephyrin (a,b) GPHN.FingR-GFP localization in a rat cortical neuron co-expressing GPHN.FingRGFP and doxycycline(dox)-inducible GFE3-TagRFP before (a) and after (b) addition of dox $(1 \mu \mathrm{g} / \mathrm{ml}$ ) for 5 hours. (c) Immunostaining of GFE3-TagRFP (red) and Gephyrin (green) in the same cell as in (a) and (b). (d) GPHN.FingR-GFP localization in a rat cortical neuron coexpressing GPHN.FingR-GFP and dox-inducible GFE3-TagRFP before (d) and after addition of dox ( $1 \mu \mathrm{g} / \mathrm{ml})$ for 24 hours (e) as well as after subsequent removal of dox for 48 hours (f). (g) An overlay of time points $t=0$ (d) and $t=72 \mathrm{hrs}$ (f). (h) Immunostaining of 
the neuron in (f) for GPHN.FingR-GFP (green), Gephyrin (red), and GAD-65 (blue) at $\mathrm{t}=$ $72 \mathrm{hrs}$. Inset: arrowheads point to puncta where all three proteins are colocalized. Scale bars represent $10 \mu \mathrm{m}$. (i) Quantitation of relative synaptic strength, by measuring total intensity of puncta labeled with GPHN.FingR-GFP, indicates that no significant difference exists between synapses at $0 \mathrm{hrs}$ and at $72 \mathrm{hrs}$ in neurons treated with dox or with vehicle $(P>0.2$, Mann-Whitney; $\mathrm{n}=7$ cells, 2 independent experiments). 